\title{
Aberrant SERCA3 expression during the colorectal adenoma-adenocarcinoma sequence
}

\author{
WEN-FENG GOU ${ }^{1}$, ZHE-FENG NIU ${ }^{1}$, SHUANG ZHAO ${ }^{1}$, YASUO TAKANO ${ }^{2}$ and HUA-CHUAN ZHENG ${ }^{1}$ \\ ${ }^{1}$ Cancer Research Center, Liaoning Medical University, Jinzhou, Liaoning 121001, P.R. China; \\ ${ }^{2}$ Clinical Research Institute, Kanagawa Cancer Center, Yokohama 241-0815, Japan
}

Received July 24, 2013; Accepted September 16, 2013

DOI: 10.3892/or.2013.2837

\begin{abstract}
Sarco/endoplasmic reticulum $\mathrm{Ca}^{2+}$-ATPase (SERCA) 3 is involved in calcium mobilization from the endoplasmic reticulum into the cytosol and is closely linked to metabolism, neuronal plasticity, gene transcription, cell growth, differentiation, apoptosis, protein folding and carcinogenesis. In order to elucidate the role of SERCA3 in colorectal carcinogenesis and subsequent progression, its expression was examined using immunohistochemistry and in situ hybridization (ISH) on tissue microarrays containing colorectal carcinomas, adjacent non-neoplastic mucosa (NNM) and adenoma, and metastatic carcinoma in lymph node and liver. Colorectal carcinoma tissue and cell lines were assessed for SERCA3 expression by western blotting or RT-PCR, respectively. SERCA3 was distinctively expressed in Colo201, Colo205, DLD-1, HCT-15, HCT-116, HT-29, KM-12, SW480, SW620 and WiDr cells at both the mRNA and protein levels. SERCA3 mRNA expression was low in carcinoma when compared to that in matched NNM by quantitative PCR $(\mathrm{P}<0.05)$, while the converse was true by ISH. Lower expression of SERCA3 was immunohistochemically observed in colorectal carcinoma when compared to that in NNM and adenoma $(\mathrm{P}<0.05)$. In contrast, primary carcinoma showed high SERCA3 expression when compared to that in metastatic carcinoma in lymph node or liver by IHC $(\mathrm{P}<0.05)$. Immunohistochemically, SERCA3 expression was negatively related to lymphatic invasion, but not with age, gender, depth of invasion, venous invasion, lymph node metastasis, distant metastasis, TNM stage, degree of differentiation or survival rate $(\mathrm{P}>0.05)$. There was a positive relationship between SERCA3 expression and serum CEA levels in the carcinoma patients $(\mathrm{P}<0.05)$. Cox's proportional hazards model indicated that depth of invasion and distant metastasis are independent prognostic factors for overall colorectal carcinomas $(\mathrm{P}<0.05)$.
\end{abstract}

Correspondence to: Professor Hua-Chuan Zheng, Cancer Research Center, Liaoning Medical University, Jinzhou, Liaoning 121001, P.R. China

E-mail: zheng_huachuan@hotmail.com

Key words: colorectal carcinoma, SERCA3, prognosis, pathogenesis, progression
These findings suggest that aberrant SERCA3 expression is closely linked to the adenoma-adenocarcinoma sequence and progression of colorectal carcinomas.

\section{Introduction}

Calcium mobilization from the endoplasmic reticulum (ER) into the cytosol is a key component of several signaling networks, including secretion, contraction, metabolism, neuronal plasticity, gene transcription, cell growth, differentiation, apoptosis or protein folding. The spatial and temporal control of intracellular $\mathrm{Ca}^{2+}$ homeostasis is crucial for regulating diverse $\mathrm{Ca}^{2+}$-dependent physiological events and is regulated by the sarco(endo)plasmic reticulum $\mathrm{Ca}^{2+}$-ATPases (SERCAs) via the hydrolysis of ATP. SERCA proteins are encoded by a family of structurally related and alternatively spliced transcripts from three distinct genes (SERCA1, SERCA2 and SERCA3) expressed in a tissue-dependent and/or developmentally regulated manner (1). Among them, SERCA3 is localized to 17 p13.3 with the encoding of 4698-bp mRNA, and is alternatively spliced to generate six different transcripts encoding the isoforms SERCA3a, SERCA3b, SERCA3c, SERCA3d, $S E R C A 3 e$ and SERCA3f under the control of the hypertension and protein kinase $\mathrm{C}$, calcineurin, or retinoic acid receptor signaling pathways. Decreased expression of SERCA3 in response to biotin supplementation by influencing the nuclear abundance of Sp1 and Sp3, suppressors of SERCA3, is likely to affect the oxidative folding of secretory proteins in the ER (2).

Although SERCA3 transcripts are most abundantly expressed in lymphoid tissues, intestine, pancreas and prostate, the highest SERCA3 expression is observed in cells of hematopoietic lineage (platelets, mast cells and lymphocytes), embryologically related endothelial cells, secretory epithelial cells (large and small intestine, thymus and trachea), and Purkinje neurons in the cerebellum. More interestingly, SERCA3 is always coexpressed with the ubiquitous isoform SERCA2b $(3,4)$. Studies using SERCA3-deficient mice have revealed that lack of SERCA3 alters the endothelium, epithelium-dependent relaxation in vascular smooth muscle and epithelium-dependent relaxation in tracheal smooth muscle $(5,6)$. Downregulation of SERCA3 expression is suggested to be responsible for the impaired glucose responses in the islets of Langerhans in diabetic mouse and rat models of non-insulin-dependent diabetes mellitus (7). In humans, 
missense mutations in the SERCA3 gene are thought to render patients more susceptible to type 2 diabetes mellitus (8). In contrast, knockout of the SERCA3 gene leads to defective endothelium-dependent relaxation of vascular smooth muscle and endothelial cell $\mathrm{Ca}^{2+}$ signaling, disruption of mixed $\left(\mathrm{Ca}^{2+}\right)$ oscillations, increased $\beta$-cell depolarization, and higher insulin response of islets to glucose $(6,9)$. It was reported that SERCA3 expression is significantly reduced or lost in colon carcinomas when compared with normal colonic epithelial cells (10). Treatment of tumor cells with butyrate or other differentiation inducing agents was found to result in a marked and specific induction of the expression of SERCA3, suggesting it constitutes a significant new differentiation marker (10). In our previous study, it was found that SERCA3 mRNA was highly expressed in gastric carcinoma when compared with that in matched non-neoplastic mucosa (NNM). SERCA3 protein expression was decreased from gastric NNM, primary to metastatic carcinoma. SERCA3 expression was also found to be negatively related to depth of invasion, distant metastasis and TNM staging of gastric carcinoma. These findings suggest that downregulated SERCA3 expression is closely linked to pathogenesis, invasion, metastasis and prognosis of gastric carcinomas (11).

Colorectal cancer is one of the most common cancers in the world, accounting for nearly $10 \%$ of newly diagnosed cases of all cancer. Japan has experienced a marked increase in the incidence of colorectal cancer, and has recently been listed among countries with the world's highest incidence rates $(12,13)$. Although pathological and genetic observations demonstrate that colorectal adenoma precedes the majority of adenocarcinoma cases, the molecular mechanisms underlying colorectal carcinogenesis are poorly understood. To investigate the roles of SERCA3 expression in colorectal carcinogenesis and subsequent progression, we examined the expression of SERCA3 mRNA and protein in colorectal NNM, adenoma, adenocarcinoma, and compared them with clinicopathological parameters of carcinomas, including serum carcinoembryonic antigen (CEA) concentration.

\section{Materials and methods}

Cell culture. The colorectal carcinoma cell lines were kindly provided by Professor Sugiyama, Department of Gastroenterology, Graduate School of Medical and Pharmaceutical Sciences, University of Toyama, Japan and Professor Miyagi, Clinical Research Institute, Kanagawa Cancer Center, Japan. They were maintained in RPMI-1640 (Colo201, Colo205, DLD-1, HCT-15, HCT-116, HT-29, KM-12, SW480 and SW620) and DMEM (WiDr) supplemented with $10 \%$ fetal bovine serum (FBS), 100 units/ml penicillin, and $100 \mu \mathrm{g} / \mathrm{ml}$ streptomycin in a humidified atmosphere of $5 \%$ $\mathrm{CO}_{2}$ at $37^{\circ} \mathrm{C}$. All cells were harvested by centrifugation, rinsed with phosphate-buffered saline (PBS) and subjected to total protein extraction by sonication in RIPA lysis buffer [50 mmol/1 Tris- $\mathrm{HCl}(\mathrm{pH} 7.5), 150 \mathrm{mmol} / \mathrm{l} \mathrm{NaCl}, 5 \mathrm{mmol} / 1$ EDTA, $0.5 \%$ Nonidet P-40, $5 \mathrm{mmol} / 1$ dithiothreitol, $10 \mathrm{mmol} / 1$ $\mathrm{NaF}$ and protease inhibitor cocktail (Sigma)].

Subjects. Colorectal carcinomas (CRCs, $n=481)$, adjacent adenoma $(n=22)$, adjacent non-neoplastic mucosa (NNM, $\mathrm{n}=475)$, metastatic foci in lymph node metastasis $(\mathrm{n}=154)$ and in liver $(n=24)$ were collected from surgical resections at the Affiliated Hospital, Kanagawa Cancer Center, Japan between 1995 and 1999. The patients with CRC included 278 men and 203 women (age range, 26-85 years; mean age, 64.1 years). Among the patients, 209 cases were accompanied by lymph node metastasis and 28 cases with liver metastasis. Thirty cases of CRCs and paired NNM were obtained from the First Affiliated Hospital of China Medical University, China, and frozen at $-80^{\circ} \mathrm{C}$ until protein and RNA extraction by homogenization. None of the patients underwent chemotherapy, radiotherapy or adjuvant therapy prior to surgery. The patients or their relatives provided consent for the use of tumor tissue for clinical research, and our University Ethics Committee approved the research protocol. We followed up the patients by reviewing their case documents or by telephone.

Pathology and tissue microarray (TMA). All tissues were fixed in $10 \%$ neutral formalin, embedded in paraffin and cut into $4 \mu \mathrm{m}$ sections. These sections were stained with hematoxylin and eosin ( $\mathrm{H} \& \mathrm{E})$ to confirm their histological characteristics. The staging for each colorectal carcinoma was evaluated according to the Union Internationale Contre le Cancer (UICC) system (14). Histological architecture of CRCs was expressed in terms of WHO classification (15). Furthermore, tumor size, depth of invasion, lymphatic and venous invasion were determined.

Representative areas of the solid tumors were identified in the H\&E-stained sections of the selected tumor cases, and a 2-mm diameter tissue core per donor block was punched out and transferred to a recipient block with a maximum of 48 cores using a tissue microarrayer (Azumaya KIN-1; Azumaya, Tokyo, Japan).

RT-PCR and DNA sequencing. Total RNA was extracted from the colorectal carcinoma cell lines or tissues using Qiagen RNeasy Mini kit (Qiagen, Hilden, Germany) according to the manufacturer's protocol. Two micrograms of total RNA was subjected to cDNA synthesis using the AMV transcriptase and random primers (Takara, Otsu, Japan). Oligonucleotide primers for PCR were 5'-AGTGCTCCGAAGACAACCC-3' and 5'-GT TCTCCGAGACGCTGTTG-3' for SERCA3 (134 bp, 2275-2908,NM_174953.1); and sense, 5'-CAATGACCCCTTC ATTGACC-3' and antisense, 5'-TGGAAGATGGTGATGGG ATT-3' for GAPDH (135 bp, 201-335, NM_002046.3). PCR amplification of cDNA was performed in 25- $\mu$ l mixtures containing $0.125 \mu \mathrm{l} \mathrm{Pfu}$ (Stratagene, West Cedar Creek, USA) with $2.0 \mathrm{mmol} \mathrm{MgCl}_{2}, 2.5 \mu \mathrm{l} 10 \mathrm{X}$ PCR buffer, $2 \mu \mathrm{ldNTP}$ mixture, $1 \mu \mathrm{mol} / 1$ of each primer set and $100 \mathrm{ng}$ of template cDNA. PCR conditions were denaturation at $95^{\circ} \mathrm{C}$ for $10 \mathrm{~min}$, followed by 35 cycles of denaturation at $95^{\circ} \mathrm{C}$ for $30 \mathrm{sec}$, annealing for $30 \mathrm{sec}$ and extension at $72^{\circ} \mathrm{C}$ for $50 \mathrm{sec}$. As a termination step, the extension time of the last cycle was increased to $7 \mathrm{~min}$. The amplicons were electrophoresed in $2 \%$ agarose gel for $30 \mathrm{~min}$. Real-time PCR was performed according to the protocol of the SYBR Premix Ex Taq ${ }^{\mathrm{TM}}$ II kit (Takara, Tokyo, Japan).

Amplicons were subjected to electrophoresis on 2\% agarose gel and purified with the QIAquick gel extraction kit (Qiagen). After extraction, the DNA was quantified by a NanoDrop 


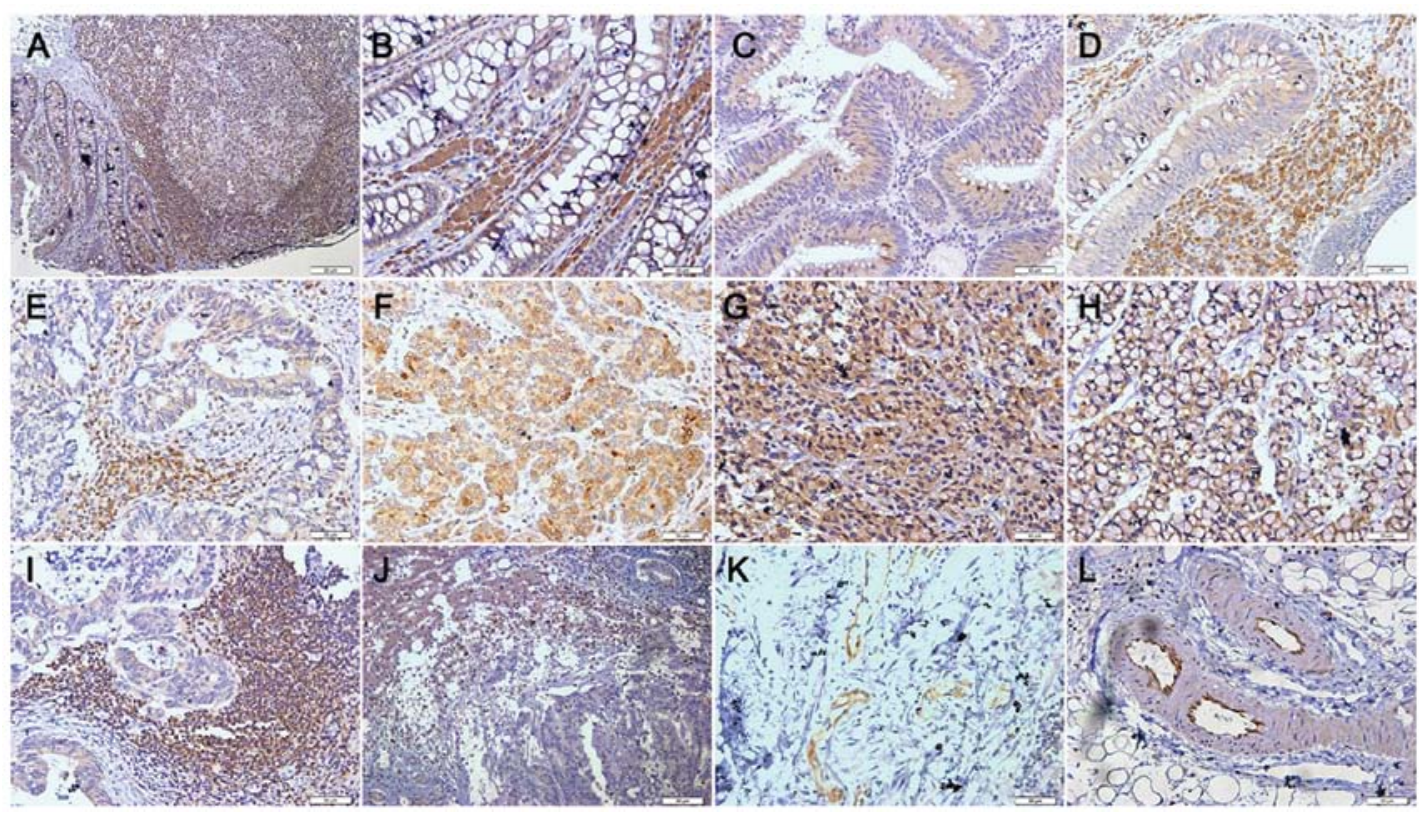

Figure 1. Immunostaining analysis of SERCA3 in colorectal lesions. SERCA3 was expressed in the (A and B) cytoplasm of the superficial epithelium, (A) infiltrating inflammatory cells, (B) macrophages, (A) lymphoid follicle, (C and D) adenoma, and (E) well-differentiated, (F) moderately-differentiated, and (G) poorly-differentiated adenocarcinoma, (H) signet ring cell carcinoma, (I) metastatic carcinoma in lymph node and (J) liver, (K) endothelial cells in vein and (L) artery.

ND-1000 spectrophotometer (Laboratory \& Medical Supplies, Tokyo, Japan) and then sequenced using a BigDye Terminator v3.1 cycle sequencing kit (Applied Biosystems, Foster City, CA, USA) and SERCA3 primers according to the recommended protocol. The sequence data were compared with the human SERCA3 cDNA sequence (NM_174953.1) using BLAST.

Western blot analysis. Denatured protein was separated on an SDS-polyacrylamide gel (10\% acrylamide) and transferred to an Amersham Hybond membrane (Amersham, Germany), which was then blocked overnight in 5\% skim milk in TBST (10 mmol/l Tris-HCl, $150 \mathrm{mmol} / \mathrm{l} \mathrm{NaCl}, 0.1 \%$ Tween-20). For immunoblotting, the membrane was incubated for $15 \mathrm{~min}$ with the mouse antibody against SERCA3 (1:200) (XL-6, sc-101265; Santa Cruz Biotechnology, Santa Cruz, CA, USA). Then, it was rinsed with TBST and incubated with anti-mouse IgG conjugated to horseradish peroxidase $(1: 1,000)$ (Dako, Carpinteria, CA, USA) for $15 \mathrm{~min}$. All of the incubations were performed in a microwave oven to allow intermittent irradiation as recommended by $\mathrm{Li}$ et al (16). Bands were visualized on X-ray film (Fujifilm, Tokyo, Japan) using ECL-Plus detection reagents (Santa Cruz Biotechnology). Finally, the membrane was washed with Western Blot Stripping Solution (pH 2.0-3.0; Nacalai, Tokyo, Japan) for $1 \mathrm{~h}$ and treated as previously described except for the anti-GAPDH antibody $(1: 10,000)$ (Sigma) as the internal control.

Immunofluorescence. Cells were grown on glass coverslips, washed twice with PBS, fixed with $4 \%$ formaldehyde for $10 \mathrm{~min}$ at room temperature and permeabilized with $0.2 \%$ Triton X-100 in PBS for $10 \mathrm{~min}$ at room temperature. After washing with PBS, cells were incubated overnight at $4^{\circ} \mathrm{C}$ with the mouse antibody against SERCA3 (1:50) (Santa Cruz Biotechnology). The cells were then washed with PBS and incubated with anti-mouse Alexa Fluor $594 \operatorname{IgG}(1: 2,000)$ (Invitrogen). Nuclei were stained using $1 \mu \mathrm{g} / \mathrm{ml}$ DAPI (Sigma) for $30 \mathrm{~min}$ at $37^{\circ} \mathrm{C}$. Finally, coverslips were mounted with SlowFade ${ }^{\circledR}$ Gold Antifade reagent (Invitrogen) and were observed under a laser confocal scanning microscope (Leica, Wetzlar, Germany).

Immunohistochemistry. Consecutive sections were deparaffinized with xylene, rehydrated with alcohol, and subjected to antigen retrieval by irradiation in target retrieval solution (TRS; Dako) for 15 min with a microwave oven (Oriental Rotor Co., Ltd., Tokyo, Japan). The sections were quenched with 3\% hydrogen peroxide in absolute methanol for $2 \mathrm{~min}$ to block endogenous peroxidase activity. Bovine serum albumin (5\%) was then applied for 5 min to prevent non-specific binding. The sections were incubated with the anti-SERCA3 antibody (1:50) (XL-6, sc-101265; Santa Cruz Biotechnology) for $15 \mathrm{~min}$, and were then treated with the anti-mouse conjugated to horseradish peroxidase (Dako) antibody for $15 \mathrm{~min}$. All of the incubations were performed in a microwave oven to allow intermittent irradiation as previously described (17). After each treatment, the slides were washed with TBST three times for $1 \mathrm{~min}$. Binding sites were visualized with 3,3'-diaminobenzidine. After counterstaining with Mayer's haematoxylin, the sections were dehydrated, cleared and mounted. Omission of the primary antibody was used as a negative control.

As indicated in Fig. 1, SERCA3 protein was positively localized in the cytoplasm. One hundred cells were randomly selected and counted from 5 representative fields of each section in a blinded manner by two independent observers (W.-F.G. and H.-C.Z.). Inconsistent data were assessed by both observers until a final consensus was reached. The positive percentage of counted cells was graded semi-quantitatively 


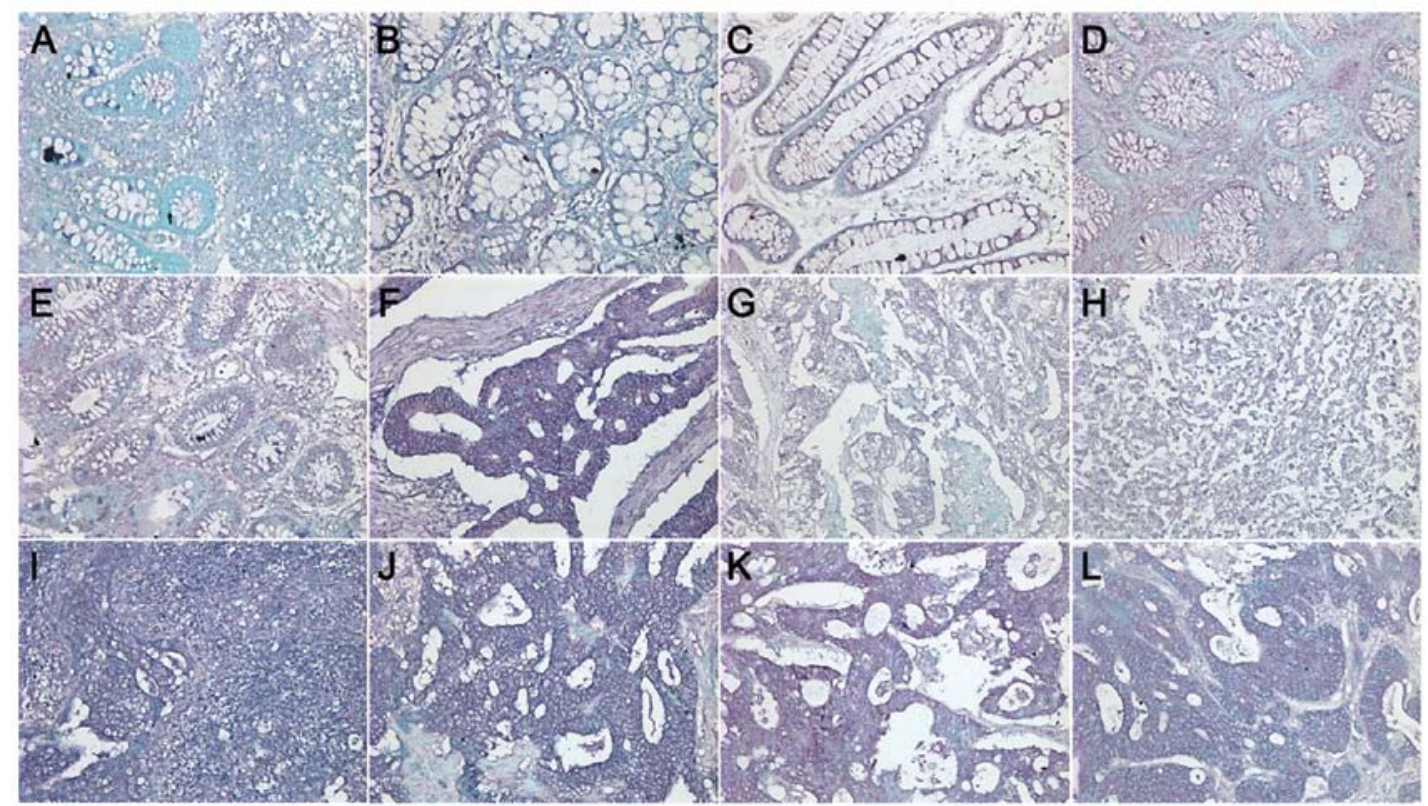

Figure 2. SERCA3 mRNA expression in colorectal mucosa, primary and metastatic carcinoma by in situ hybridization. SERCA3 mRNA was observed in the cytoplasm of the lymphocytes in (A) lymphatic follicle, (B and C) epithelial cells, (D and E) adenoma, (B-E) infiltrating inflammatory cells, (F-H) primary adenocarcinoma, (I and $\mathrm{J}$ ) metastatic carcinoma in lymph node and $(\mathrm{K}$ and $\mathrm{L})$ liver.

according to a 4-tier scoring system: negative (-), 0-5\%; weakly positive $(+), 6-25 \%$; moderately positive $(++), 26-50 \%$; and strongly positive $(+++),>50 \%$.

In situ hybridization. To perform RNA-DNA in situ hybridization (ISH) for SERCA3, a digoxygenin-labeled SERCA3 probe was constructed in 35-cycle PCR using the above-mentioned primers and $50 \mathrm{ng}$ template DNA of 30-cycle products from the DLD-1 cDNA using Pfu polymerase (Stratagene, La Jolla, CA, USA). Sections (4- $\mu \mathrm{m})$ were deparaffinized and digested with $20 \mu \mathrm{g} / \mathrm{ml}$ proteinase $\mathrm{K}$ in $50 \mathrm{mmol} / 1 \mathrm{Tris}-\mathrm{HCl}$ at $37^{\circ} \mathrm{C}$ for $10 \mathrm{~min}$. Subsequently, $20 \mu \mathrm{l}$ of a 1:20 probe dilution in hybridization buffer $(22 \mathrm{mmol} / \mathrm{l}$ Tris- $\mathrm{HCl}, \mathrm{pH}$ 7.4, $2.75 \mathrm{mmol} / \mathrm{l}$ ethylenediaminetetraacetic acid, $660 \mathrm{mmol} / 1 \mathrm{NaCl}, 1 \mathrm{X}$ Denhardt solution, $5.5 \%$ dextran sulfate, $0.33 \%$ dimethyl sulfoxide, $0.55 \%$ Ethoquad $^{\circledR} 18 / 25$ and $44 \%$ deionized formamide) was added to each slide. After coverslipping, heating at $95^{\circ} \mathrm{C}$ for $5 \mathrm{~min}$, and incubation overnight in a humidified chamber at $37^{\circ} \mathrm{C}$, sections were rinsed for $10 \mathrm{~min}$ in TBST and incubated with anti-digoxygenin antibody conjugated with alkaline phosphatase (AP; Roche Diagnostics, GmbH, Penzberg, Germany) for $20 \mathrm{~min}$ at $37^{\circ} \mathrm{C}$. The slides were then washed for $5 \mathrm{~min}$ and immersed in solution II (100 mmol/1 Tris- $\mathrm{HCl}, \mathrm{pH} \mathrm{9.5,}$ $100 \mathrm{mmol} / 1 \mathrm{NaCl}$ and $50 \mathrm{mmol} / 1 \mathrm{MgCl}_{2}$ ) for $30 \mathrm{~min}$ followed by exposure to NBT (Nitroblue tetrazolium chloride)/BCIP (5-bromo-4-chloro-3'-indolyl phosphatase $p$-toluidine salt) as a chromogen. Finally, counter staining was performed using methyl green for $2 \mathrm{~min}$.

As indicated in Fig. 2, the mRNA signal of SERCA3 was positively localized in the cytoplasm. One hundred cells were randomly selected and counted from 5 representative fields of each section in a blinded manner by two independent observers (W.-F.G. and H.-C.Z.). Inconsistent data were assessed by both observers until a final consensus was reached. The scoring system was the same as that for immunohistochemistry.
Measurement of carcinoembryonic antigen (CEA). Serum CEA was determined using a chemiluminescence immunoassay (Diagnostic Agnostic Automation Inc.). Briefly, $50 \mu \mathrm{l}$ of standard $(0-120 \mathrm{ng} / \mathrm{ml})$, specimens and controls was dispensed into appropriate wells. We then added $100 \mu \mathrm{l}$ of the enzyme conjugate reagent into each well, gently mixed and incubated the plate at room temperature for $60 \mathrm{~min}$. The microtiter wells were rinsed and flicked with wash buffer. After that, residual water droplets were removed by striking the well sharply onto absorbent paper. Finally, $100 \mu \mathrm{l}$ chemiluminescence substrate solution into each well was dispensed, mixed gently and subjected to determination of absorbance.

Statistical analysis. Statistical analysis was performed using the Spearman correlation test to analyze the rank data, and the Mann-Whitney $U$ test to differentiate the means of the different groups. Kaplan-Meier survival plots were generated, and comparisons were carried out with log-rank statistics. Cox's proportional hazards model was employed for multivariate analysis. $\mathrm{P}<0.05$ was considered to indicate a statistically significant result. SPSS 10.0 software was employed to analyze all data.

\section{Results}

SERCA3 expression in colorectal tumors and carcinoma cell lines. SERCA3 protein was detected in the cytoplasm of all colorectal carcinoma cell lines by western blot analysis and immunofluorescence (Fig. 3A and C). To assess SERCA3 mRNA expression, we employed RT-PCR. SERCA3 mRNA was expressed at equal levels in these carcinoma cell lines (Fig. 3B). Additionally, all of the amplicons were subjected to direct DNA sequencing and confirmed to be accurate (data not shown). Among 27 frozen samples of colorectal carcinoma, decreased SERCA3 mRNA expression was observed in 24 

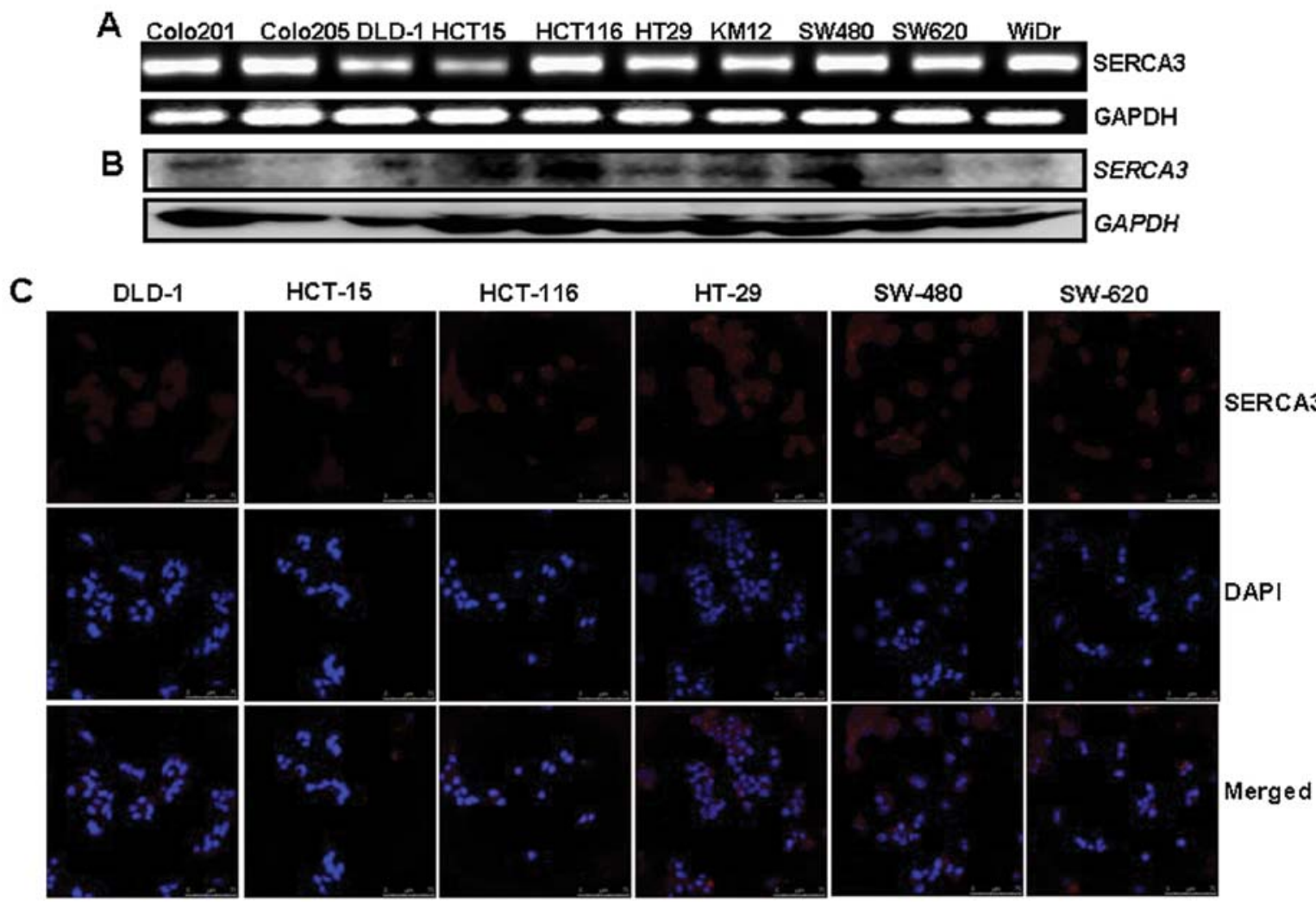

Figure 3. SERCA3 expression in colorectal carcinoma cell lines. (A) Distinct expression of SERCA3 mRNA (120 bp) was detected and showed consistent density in all colorectal carcinoma cell lines with an internal control of GAPDH (135 bp). (B) Tissue lysate was loaded and probed with the anti-SERCA3 antibody $(97 \mathrm{kDa})$ with GAPDH ( $36 \mathrm{kDa}$ ) as an internal control. (C) All colorectal carcinoma and epithelial carcinoma cell lines were subjected to immunofluorescence staining of SERCA3 protein (red, SERCA3; blue, DAPI).
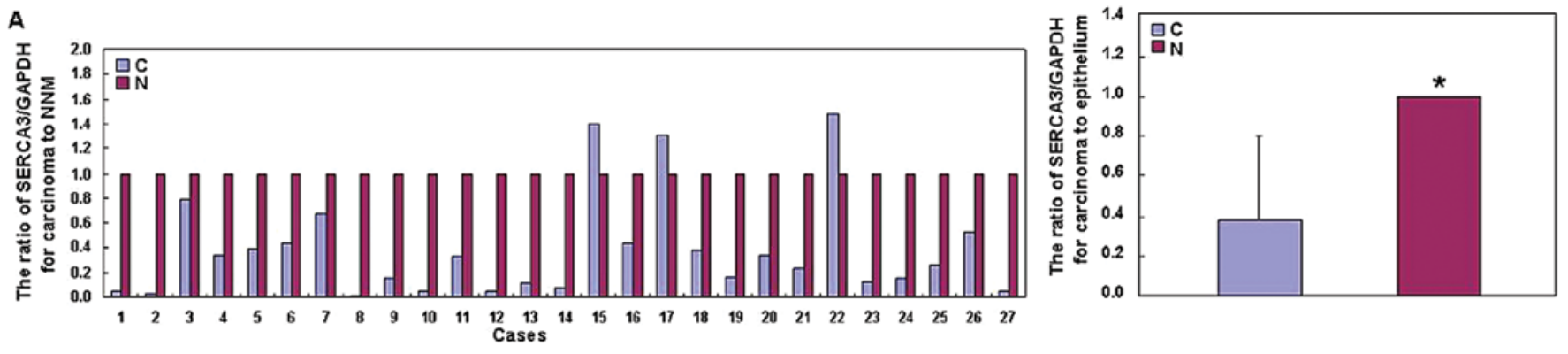

B
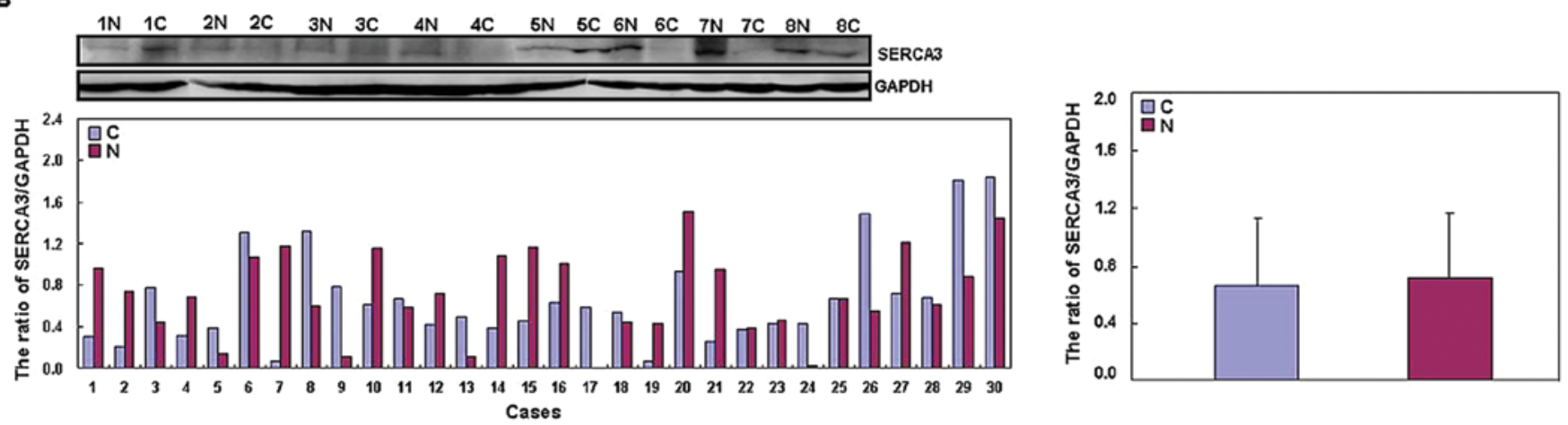

Figure 4. SERCA3 expression in colorectal carcinoma cases and matched mucosa. (A) The SERCA3 mRNA expression level was lower in the colorectal carcinoma cases than that in the paired mucosa by real-time RT-PCR $(\mathrm{P}<0.05)$. (B) Tissue lysate was loaded and probed with the anti-SERCA3 antibody $(97 \mathrm{kDa})$ with GAPDH (36 kDa) used as an internal control. Densitometry analysis of the SERCA3 bands showed no difference in the SERCA3 protein expression level between the colorectal carcinoma and the paired non-neoplastic mucosa $(\mathrm{P}>0.05)$. N, non-neoplastic mucosa; $\mathrm{C}$, carcinoma. 
Table I. SERCA3 expression in colorectal non-neoplastic mucosa, adenomas and carcinomas.

\begin{tabular}{|c|c|c|c|c|c|c|}
\hline \multirow[b]{2}{*}{ Groups } & \multirow[b]{2}{*}{$\mathrm{n}$} & \multicolumn{4}{|c|}{ SERCA3 expression } & \multirow[b]{2}{*}{$\mathrm{PR}(\%)$} \\
\hline & & - & + & ++ & +++ & \\
\hline NNMs & 475 & 32 & 37 & 121 & 285 & 93.3 \\
\hline Adenomas adjacent to carcinoma & 22 & 1 & 1 & 7 & 13 & 95.5 \\
\hline Primary carcinoma & 481 & 236 & 85 & 91 & 69 & $50.8^{\mathrm{a}}$ \\
\hline Metastatic carcinoma in lymph node & 154 & 104 & 24 & 17 & 9 & $32.5^{\mathrm{b}}$ \\
\hline Metastatic carcinoma in liver & 24 & 17 & 5 & 2 & 0 & $29.2^{\mathrm{c}}$ \\
\hline
\end{tabular}

PR, positive rate; NNM, non-neoplastic mucosa. ${ }^{\mathrm{a} C o m p a r e d ~ w i t h ~ N N M ~ o r ~ a d e n o m a s, ~} \mathrm{P}<0.001$. ${ }^{\mathrm{b}}$ Compared with primary carcinoma, $\mathrm{P}<0.001$. ${ }^{\mathrm{c}}$ Compared with primary carcinoma, $\mathrm{P}=0.011$.

Table II. SERCA3 mRNA expression in colorectal non-neoplastic mucosa, adenomas and carcinomas.

\begin{tabular}{|c|c|c|c|c|c|c|}
\hline \multirow[b]{2}{*}{ Groups } & \multirow[b]{2}{*}{$\mathrm{n}$} & \multicolumn{4}{|c|}{$S E R C A 3$ expression } & \multirow[b]{2}{*}{$\operatorname{PR}(\%)$} \\
\hline & & - & + & ++ & +++ & \\
\hline NNMs & 76 & 39 & 15 & 12 & 10 & 48.7 \\
\hline Adenomas adjacent to carcinoma & 93 & 40 & 13 & 18 & 22 & 57.0 \\
\hline Primary carcinoma & 111 & 40 & 27 & 22 & 22 & $64.0^{\mathrm{a}}$ \\
\hline Metastatic carcinoma in lymph node & 46 & 8 & 2 & 18 & 18 & 82.6 \\
\hline Metastatic carcinoma in liver & 26 & 9 & 3 & 2 & 12 & 65.4 \\
\hline
\end{tabular}

PR, positive rate; NNM, non-neoplastic mucosa. ${ }^{\mathrm{a} C o m p a r e d}$ with NNM, and metastatic carcinomas in lymph node, $\mathrm{P}<0.05$.

cases $(88.9 \%)$ when compared with the mRNA expression in the adjacent mucosa by real-time PCR (Fig. 4A). SERCA3 mRNA expression was low in the carcinoma samples than that in the matched NNM $(\mathrm{P}<0.05)$. However, there was no difference in SERCA3 expression between carcinoma and paired NNM by western blot analysis ( $\mathrm{P}>0.05$; Fig. 4B).

As shown in Fig. 1, SERCA3 was expressed in the cytoplasm of the superficial mucosa, infiltrating inflammatory cells, macrophages, lymphoid follicle, adenoma, well-differentiated, moderately differentiated and poorly differentiated adenocarcinoma, signet ring cell carcinoma, metastatic carcinoma in lymph node and liver, and endothelial cells in the vein and artery. SERCA3 expression was detected in $93.3 \%$ $(443 / 475)$ of the NNM, 95.5\% (21/22) of adjacent adenomas, $50.8 \%(245 / 482)$ of primary carcinomas, $32.5 \%(50 / 154)$ of the metastatic carcinomas in lymph node and $29.2 \%(7 / 24)$ of metastatic carcinomas in the liver. When statistics was performed, we considered the 4-graded semi-quantitative scoring and employed Spearman correlation analysis. Low expression of SERCA3 was observed in the colorectal carcinoma cases when compared to that in the NNM and adenomas $(\mathrm{P}<0.05$; Table I). In contrast, primary carcinoma showed higher SERCA3 expression than metastatic carcinomas in the lymph node or liver $(\mathrm{P}<0.05$; Table I).

To confirm SERCA3 mRNA expression, in situ hybridization(ISH) was also employed on TMAs of colorectal carcinoma, NNM, adenoma, primary and metastatic carcinoma (Fig. 2). The positive signal of SERCA3 mRNA was detectable in the cytoplasm of colorectal epithelial cells, adenoma, carcinoma, infiltrating inflammatory cells or lymphocytes in lymphatic follicles. SERCA3 mRNA was detected in colorectal NNM $(48.7 \%, 37 / 76)$, adenoma $(57.0 \%, 53 / 93)$, primary carcinoma $(64.0 \%, 71 / 111)$, metastatic carcinoma in lymph node $(82.6 \%$, $38 / 46)$ and liver $(65.4 \%, 17 / 26)$. Statistically, its expression was lower in NNM than that in the colorectal carcinoma $(\mathrm{P}<0.05$; Table II). SERCA3 mRNA was expressed at a lower level in the primary carcinoma when compared to that in the metastatic carcinoma in lymph node $(\mathrm{P}<0.05$; Table II).

Association of SERCA3 expression with clinicopathological parameters of the colorectal carcinoma cases. As shown in Table III, SERCA3 expression was negatively correlated with lymphatic invasion $(\mathrm{P}<0.05)$, but not with age, gender, depth of invasion, venous invasion, lymph node metastasis, distant metastasis, TNM stage or degree of differentiation $(\mathrm{P}>0.05)$. The serum CEA concentration was higher in the carcinoma patients with positive SERCA3 expression than that in patients without SERCA3 positivity $(\mathrm{P}<0.05$; Fig. 5). Follow-up information was available for 381 colorectal carcinoma patients for a period ranging from 1.6 months to 16.8 years (median, 10.5 years). Survival curves for colorectal carcinomas were stratified according to SERCA3 expression (Fig. 6). Univariate analysis using the Kaplan-Meier method indicated that the cumulative survival rate of patients was not linked to SERCA3 expression status $(\mathrm{P}>0.05)$, even when the data were stratified according to the depth of invasion (data not shown). Multivariate analysis 
Table III. Relationship between SERCA3 expression and clinicopathological features of the CRC cases.

\begin{tabular}{|c|c|c|c|c|c|c|c|}
\hline \multirow{2}{*}{$\begin{array}{l}\text { Clinicopathological } \\
\text { features }\end{array}$} & \multirow[b]{2}{*}{$\mathrm{n}$} & \multicolumn{4}{|c|}{ SERCA3 expression } & \multirow[b]{2}{*}{$\mathrm{PR}(\%)$} & \multirow[b]{2}{*}{$\mathrm{P}$-value } \\
\hline & & - & + & ++ & +++ & & \\
\hline Age (years) & & & & & & & 0.941 \\
\hline$<65$ & 260 & 126 & 48 & 50 & 36 & 51.5 & \\
\hline$\geq 65$ & 221 & 110 & 37 & 41 & 33 & 50.2 & \\
\hline Gender & & & & & & & 0.865 \\
\hline Male & 278 & 134 & 56 & 51 & 37 & 51.8 & \\
\hline Female & 203 & 102 & 29 & 40 & 32 & 49.8 & \\
\hline Depth of invasion & & & & & & & 0.641 \\
\hline $\mathrm{T}_{\mathrm{is}}-\mathrm{T}_{2}$ & 123 & 58 & 23 & 22 & 20 & 52.8 & \\
\hline $\mathrm{T}_{3}-\mathrm{T}_{4}$ & 338 & 168 & 58 & 63 & 49 & 50.3 & \\
\hline Lymphatic invasion & & & & & & & 0.015 \\
\hline- & 195 & 82 & 38 & 39 & 36 & 57.9 & \\
\hline+ & 207 & 112 & 32 & 38 & 25 & 45.9 & \\
\hline Venous invasion & & & & & & & 0.473 \\
\hline- & 193 & 99 & 33 & 30 & 31 & 48.7 & \\
\hline+ & 196 & 92 & 33 & 43 & 28 & 53.1 & \\
\hline Lymph node metastasis & & & & & & & 0.211 \\
\hline- & 253 & 119 & 43 & 50 & 41 & 53.0 & \\
\hline+ & 209 & 108 & 37 & 38 & 26 & 48.3 & \\
\hline Distant metastasis & & & & & & & 0.895 \\
\hline- & 441 & 216 & 76 & 84 & 65 & 51.0 & \\
\hline+ & 36 & 17 & 8 & 7 & 4 & 52.8 & \\
\hline TNM stage & & & & & & & 0.424 \\
\hline I-II & 242 & 116 & 42 & 44 & 40 & 52.1 & \\
\hline III-IV & 217 & 109 & 38 & 43 & 27 & 49.8 & \\
\hline Differentiation & & & & & & & 0.136 \\
\hline Well-differentiated & 194 & 88 & 32 & 38 & 36 & 54.6 & \\
\hline Moderately-differentiated & 231 & 118 & 44 & 45 & 24 & 48.9 & \\
\hline Poorly-differentiated & 32 & 16 & 5 & 4 & 7 & 50.0 & \\
\hline
\end{tabular}

$\mathrm{CRC}$, colorectal carcinoma; $\mathrm{PR}$, positive rate; $\mathrm{T}_{\mathrm{is}}$, carcinoma in situ; $\mathrm{T}_{1}$, lamina propria and submucosa; $\mathrm{T}_{2}$, muscularis propria; $\mathrm{T}_{3}$, subserosa and exposure to serosa; $\mathrm{T}_{4}$, invasion of other organs or perforate visceral peritoneum.

using Cox's proportional hazard model indicated that depth of invasion and distant metastasis $(\mathrm{P}<0.05)$, but not age, gender, lymphatic and venous invasion, lymph node metastasis, liver metastasis, differentiation, TNM stage or SERCA3 expression ( $>$ >0.05; Table IV) are independent prognostic factors for overall survival of the colorectal carcinoma parients.

\section{Discussion}

Endoplasmic reticulum calcium homeostasis is involved in a multitude of intracellular signaling, as well as 'housekeeping' functions that control cell growth, differentiation, protein synthesis or apoptosis in eukaryotic cells. In several cell systems, SERCA3 expression is selectively induced during differentiation, whereas SERCA3 expression is decreased during tumorigenesis and blastic transformation $(1,2)$. In the present study, we, for the first time, examined in situ SERCA3 expression in a large number of colorectal mucosal and carcinoma samples. It was found that SERCA3 protein was mainly localized in the cytoplasm of the superficial epithelium, infiltrating inflammatory cells, macrophages, lymphoid follicle, adenoma, adenocarcinoma, signet ring cell carcinoma, and endothelial cells in vein and artery. It was suggested that the SERCA3 expression pattern has cellular specificity, which determines its biological functions. However, the mechanisms of its cell-specific characteristics warrant further investigation.

To clarify the in situ expression pattern and the clinicopathological significance of the SERCA3 protein, IHC and ISH were performed on TMAs of colorectal lesions. Statistically, SERCA3 expression was reduced in the colorectal carcinoma, when compared with that in the adjacent non-neoplastic mucosa and adenoma in line with previous reports $(11,18)$. Real-time 
Table IV. Multivariate analysis of the clinicopathological variables for the overal survival of the CRC patients.

\begin{tabular}{llr}
\hline Clinicopathological parameters & Relative risk (95\% CI) & P-value \\
\hline Age ( $\geq 65$ years) & $0.875(0.553-1.384)$ & 0.567 \\
Gender (female) & $0.850(0.544-1.327)$ & 0.474 \\
Depth of invasion $\left(\mathrm{T}_{2-4}\right)$ & $8.027(2.439-26.424)$ & 0.001 \\
Lymphatic invasion (+) & $1.242(0.729-2.116)$ & 0.426 \\
Venous invasion (+) & $1.017(0.598-1.731)$ & 0.950 \\
Lymph node metastasis $(+)$ & $1.810(0.549-5.961)$ & 0.329 \\
Distant metastasis & $4.826(2.292-10.162)$ & $<0.001$ \\
UICC staging (I-II) & $1.657(0.439-6.257)$ & 0.456 \\
Differentiation (moderately and poorly) & $1.129(0.790-1.614)$ & 0.505 \\
SERCA3 expression (+-+++) & $1.076(0.681-1.701)$ & 0.754
\end{tabular}

CRC, colorectal carcinoma; CI, confidence interval; UICC, Union Internationale Contre le Cancer.

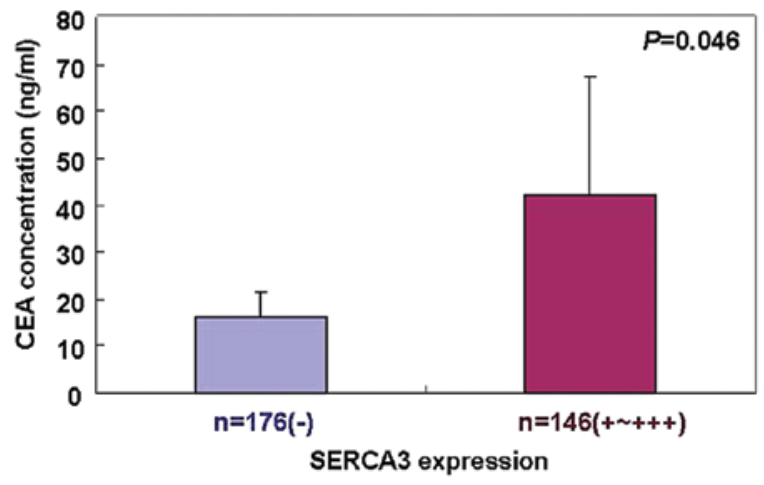

Figure 5. Relationship between SERCA3 expression and the serum CEA level in the colorectal carcinoma patients. The serum CEA concentration was high in the carcinoma patients with positive SERCA3 expression when compared with the concentration in patients without SERCA3 positivity $(\mathrm{P}<0.05)$.

PCR showed that SERCA3 mRNA was weakly expressed in carcinoma in comparison with the adjacent NNM. The data indicated that downregulation of SERCA3 expression may contribute to the malignant transformation of colorectal epithelial cells as a late event and its transcript expression as an early event. In our frozen samples, we found no difference in SERCA3 expression between most of the colorectal carcinoma and the matched mucosa. The paradoxical phenomenon may be explained by the different detecting approaches due to the presence of SERCA3 expression in stromal cells from colorectal samples, which were excluded from morphological data by virtue of their specific histomorphological features and topographic location in the tissue sections. The short half life of SERCA3 might be due to its downregulated expression in colorectal carcinoma possibly via proteosome-mediated degradation. Strangely, higher SERCA3 mRNA expression was detected in the carcinoma by ISH, when compared with that in the adjacent NNM, which may be due to different sample treatment and detection approaches. Brouland et al (18) reported that SERCA3 expression was increased along the crypts as cells differentiated in normal colonic mucosa and in hyperplastic polyps; was moderately and heterogeneously expressed

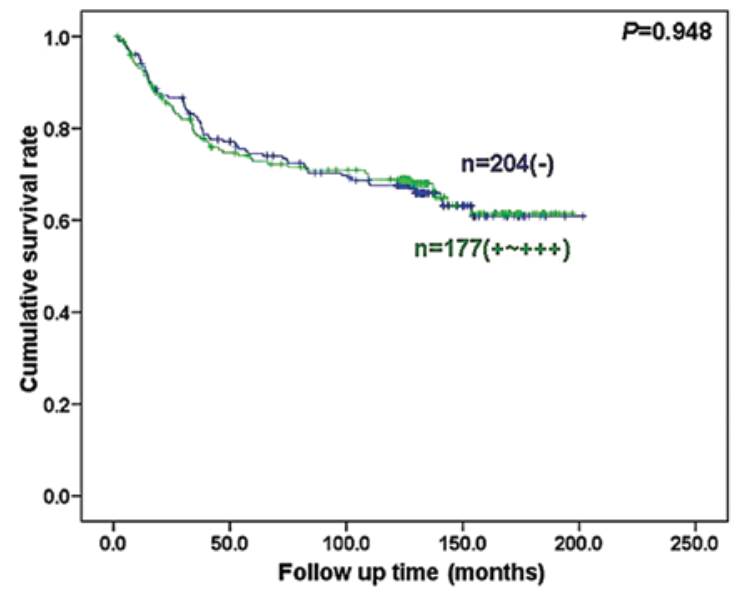

Figure 6. Correlation between the SERCA3 expression status and prognosis of the colorectal carcinoma patients. Kaplan-Meier curves for the cumulative survival rate of the colorectal carcinoma patients according to the SERCA3 expression status.

in colonic adenomas with expression levels inversely correlated with the degree of dysplasia; and was barely detectable in adenocarcinomas. During the multi-step process of colon carcinogenesis, the decrease in SERCA3 expression appears to be linked to enhanced APC/ $\beta$-catenin/TCF4 signaling and deficient Sp1-like factor-dependent transcription $(19,20)$.

In the present study, SERCA3 expression was lower in the metastatic carcinomas in the liver and lymph node, and the cases with lymphatic invasion although it was not linked to aggressive behaviors of the colorectal carcinomas, such as invasion, metastasis and TNM stage. In our previous report, SERCA3 expression was inversely correlated with depth of invasion, distant metastasis and TNM stage of gastric cancer. Combined with these findings, we believe that SERCA3 expression may be involved in the development of gastrointestinal carcinomas. Previously, we reported that SERCA3 expression was positively linked to the favorable prognosis of gastric carcinoma (11). However, this relationship did not exist for the patients with colorectal carcinoma, which might be explained by the absence of a correlation of its expression 
with aggressive behaviors. Multivariate analysis demonstrated that the depth of invasion and distant metastasis are independent prognostic factors for overall survival of the colorectal carcinoma patients.

Brouland et al (18) found that treatment of gastric or colorectal carcinoma cells with butyrate or other established differentiation-inducing agents resulted in a marked induction in the expression of SERCA3 and higher resting cytosolic calcium concentrations, suggesting that SERCA 3 constitutes a significant new differentiation marker that may prove useful for the analysis of the phenotype of gastrointestinal adenocarcinomas. However, no relationship between SERCA3 expression and the degree of differentiation of colorectal carcinoma was found in the present study although the depolarization of SERCA3 was observed with worse differentiation. This result may be due to the small number of poorly-differentiated carcinoma cases. In agreement with the present finding, there was no difference in SERCA3 expression between intestinal- and diffuse-type carcinoma and even between intestinal and diffuse components of mixed-type carcinoma of the stomach (11).

CEA is a glycosyl phosphatidyl inositol (GPI)-cell surface anchored glycoprotein and serves as functional colon carcinoma L-selectin and E-selectin ligands. It was found that serum from individuals with gastric, pancreatic, lung, breast and medullary thyroid carcinoma had higher levels of CEA than healthy individuals $(>2.5 \mathrm{ng} / \mathrm{ml})$. In colorectal carcinoma, CEA might be employed to predict early progression and worse prognosis (21-23). Reportedly, as little as $1 \%$ serum admixed with tumor cells results in a CEA release up to $200 \%$ greater than that of serum-free controls, which is dependent on calcium (24). Since SERCA3 is involved in calcium mobility of the endoplasmic reticulum, the positive link between SERCA3 expression and serum CEA level is rational in patients with colorectal carcinoma. However, further investigation of how SERCA3 regulates CEA secretion in malignancies is warranted.

In summary, the present study revealed that aberrant SERCA3 expression impacts the malignant transformation of colorectal epithelial cells. Nevertheless, the biological functions of SERCA3 in colorectal carcinomas need further investigation.

\section{Acknowledgements}

The present study was supported by the Shenyang Outstanding Talent Foundation of China; the Shenyang Science and Technology Grant (F11-264-1-10 and F12-277-1-01); the Natural Scientific Foundation of China (nos. 81172371 and 81201886); and the grant-in aid for the Scientific Research from the Ministry of Education, Culture, Sports and Technology of Japan (23659958).

\section{References}

1. Hovnanian A: SERCA pumps and human diseases. Subcell Biochem 45: 337-363, 2007.

2. Wuytack F, Dode L, Baba-Aissa F, et al: The SERCA3-type of organellar $\mathrm{Ca}^{2+}$ pumps. Biosci Rep 15: 299-306, 1995.

3. Baba-Aïssa F, Raeymaekers L, Wuytack F, et al: Purkinje neurons express the SERCA3 isoform of the organellar type $\mathrm{Ca}(2+)$-transport ATPase. Brain Res Mol Brain Res 41: 169-174, 1996.
4. Chandrasekera PC, Kargacin ME, Deans JP, et al: Determination of apparent calcium affinity for endogenously expressed human sarco(endo)plasmic reticulum calcium-ATPase isoform SERCA3. Am J Physiol Cell Physiol 296: C1105-C1114, 2009.

5. Kao J, Fortner CN, Liu LH, et al: Ablation of the SERCA3 gene alters epithelium-dependent relaxation in mouse tracheal smooth muscle. Am J Physiol Lung Cell Mol Physiol 277: L264-L270, 1999.

6. Liu LH, Paul RJ, Sutliff RL, et al: Defective endotheliumdependent relaxation of vascular smooth muscle and endothelial cell $\mathrm{Ca}^{2+}$ signalling in mice lacking sarco(endo) plasmic reticulum $\mathrm{Ca}^{2+}$-ATPase isoform 3. J Biol Chem 272: 30538-30545, 1997.

7. Roe MW, Philipson LH, Frangakis CJ, et al: Defective glucosedependent endoplasmic reticulum $\mathrm{Ca}^{2+}$ sequestration in diabetic mouse islets of Langerhans. J Biol Chem 269: 18279-18282, 1994.

8. Varadi A, Lebel L, Hashim Y, et al: Sequence variants of the sarco(endo)plasmic reticulum $\mathrm{Ca}^{2+}$-transport ATPase 3 gene (SERCA3) in Caucasian type II diabetic patients (UK Prospective Diabetes Study 48). Diabetologia 42: 1240-1243, 1999.

9. Arredouani A, Guiot Y, Jonas JC, et al: SERCA3 ablation does not impair insulin secretion but suggests distinct roles of different sarcoendoplasmic reticulum $\mathrm{Ca}^{2+}$ pumps for $\mathrm{Ca}^{2+}$ homeostasis in pancreatic $\beta$-cells. Diabetes 51: 3245-3253, 2002.

10. Gélébart P, Kovács T, Brouland JP, et al: Expression of endomembrane calcium pumps in colon and gastric cancer cells. Induction of SERCA3 expression during differentiation. J Biol Chem 277: 26310-26320, 2002.

11. Xu XY, Yang X, Takahashi H, et al: The down-regulated SERCA3 expression is closely linked to pathogenesis, invasion, metastasis and poor prognosis of gastric carcinomas. Tumour Biol 33: 1845-1854, 2012.

12. Colorectal Cancer Facts and Figures. American Cancer Society, 2005.

13. Yoshida D, Kono S, Moore MA, et al: Colorectal polypectomy and risk of colorectal cancer by subsite: the Fukuoka Colorectal Cancer study. Jpn J Clin Oncol 37: 597-602, 2007.

14. Hamilton SR and Aaltonen LA: WHO Classification of Tumors: Pathology and Genetics of Tumors of the Digestive System. IARC Press, Lyon, 2000.

15. Sobin LH and Wittekind CH: TNM Classification of Malignant Tumours. 6th edition. John Wiley \& Sons, Hoboken, NJ, 2002.

16. Li W, Murai Y, Okada E, et al: Modified and simplified western blotting protocol: use of intermittent microwave irradiation (IMWI) and 5\% skim milk to improve binding specificity. Pathol Int 52: 234-238, 2002.

17. Kumada T, Tsuneyama $\mathrm{K}$, Hatta $\mathrm{H}$, Ishizawa $\mathrm{S}$, et al: Improved 1-h rapid immunostaining method using intermittent microwave irradiation: practicability based on 5 years application in Toyama Medical and Pharmaceutical University Hospital. Mod Pathol 17: 1141-1149, 2004.

18. Brouland JP, Gélébart $\mathrm{P}$, Kovàcs $\mathrm{T}$, et al: The loss of sarco/endoplasmic reticulum calcium transport ATPase 3 expression is an early event during the multistep process of colon carcinogenesis. Am J Pathol 167: 233-242, 2005.

19. Arbabian A, Brouland JP, Gélébart P, et al: Endoplasmic reticulum calcium pumps and cancer. Biofactors 37: 139-149, 2011.

20. Brouland JP, Valleur P and Papp B: Expression of SERCA pumps during cell differentiation and tumorigenesis: application to colonic carcinogenesis. Ann Pathol 26: 159-172, 2006.

21. Petrioli R, Licchetta A, Roviello G, et al: CEA and CA19.9 as early predictors of progression in advanced/metastatic colorectal cancer patients receiving oxaliplatin-based chemotherapy and bevacizumab. Cancer Invest 3: 65-71, 2012.

22. Lan YT, Lin JK, Lin TC, et al: Prognostic significance of perioperative change of CEA level in colorectal patients when pre-operative level is normal. Hepatogastroenterology 59: 717-720, 2012.

23. Lin JK, Lin CC, Yang SH, et al: Early postoperative CEA level is a better prognostic indicator than is preoperative CEA level in predicting prognosis of patients with curable colorectal cancer. Int J Colorectal Dis 26: 1135-1141, 2011.

24. Yeatman TJ, Duan C, Mao W, et al: Augmentation of carcinoembryonic antigen release from intact, viable tumor cells by a factor in human serum. Ann Surg Oncol 2: 336-342, 1995. 